\title{
An Integrated Insight into the Relationship between Soil Microbial Community and Tobacco Bacterial Wilt Disease
}

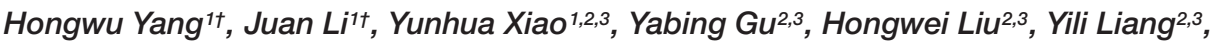 \\ Xueduan $\mathrm{Liu}^{2,3}$, Jin Hu${ }^{2,3}$, Delong Meng ${ }^{2,3 *}$ and Huaqun Yin ${ }^{2,3 *}$ \\ College of Agronomy, Hunan Agricultural University, Changsha, China, ${ }^{2}$ School of Minerals Processing and Bioengineering, \\ Central South University, Changsha, China, ${ }^{3}$ Key laboratory of Biometallurgy, Ministry of Education, Changsha, China
}

\section{OPEN ACCESS}

Edited by:

Stéphane Hacquard,

Max Planck Institute for Plant Breeding Research (MPG), Germany

Reviewed by:

Matthew Agler

Friedrich-Schiller-Universität Jena,

Germany

Ravin Poudel,

University of Florida, United States

Zhili He,

University of Oklahoma, United States

*Correspondence:

Huaqun Yin

yinhuaqun_cs@sina.com; yinhuaqun@gmail.com

Delong Meng

delong.meng@gmail.com

tThese authors have contributed equally to this work.

Specialty section:

This article was submitted to

Plant Microbe Interactions, a section of the journa

Frontiers in Microbiology

Received: 26 June 2017

Accepted: 24 October 2017

Published: 07 November 2017

Citation:

Yang $H$, Li J, Xiao Y, Gu Y, Liu H, Liang Y, Liu X, Hu J, Meng D and Yin $H$ (2017) An Integrated Insight into the Relationship between Soil Microbial Community and Tobacco Bacterial Wilt Disease.

Front. Microbiol. 8:2179.

doi: 10.3389/fmicb.2017.02179
The soil microbial communities play an important role in plant health, however, the relationship between the below-ground microbiome and above-ground plant health remains unclear. To reveal such a relationship, we analyzed soil microbial communities through sequencing of $16 \mathrm{~S}$ rRNA gene amplicons from 15 different tobacco fields with different levels of wilt disease in the central south part of China. We found that plant health was related to the soil microbial diversity as plants may benefit from the diverse microbial communities. Also, those 15 fields were grouped into 'healthy' and 'infected' samples based upon soil microbial community composition analyses such as unweighted paired-group method with arithmetic means (UPGMA) and principle component analysis, and furthermore, molecular ecological network analysis indicated that some potential plant-beneficial microbial groups, e.g., Bacillus and Actinobacteria could act as network key taxa, thus reducing the chance of plant soil-borne pathogen invasion. In addition, we propose that a more complex soil ecology network may help suppress tobacco wilt, which was also consistent with highly diversity and composition with plant-beneficial microbial groups. This study provides new insights into our understanding the relationship between the soil microbiome and plant health.

Keywords: tobacco wilt disease, plant health, soil microbial community, microbial community composition, molecular ecology networks

\section{INTRODUCTION}

Soil borne pathogens attack crops and causing huge yield losses. Bacterial wilt disease, caused by Ralstonia, can infect Solanaceae crops (e.g., tobacco, tomato, egg plants, etc.) with large scale crop damage world wide, and many efforts have been made to control this disease (Janvier et al., 2007). A number of factors have an effect on soil borne plant pathogen infections including the soil microbiome (Pieterse and Dicke, 2007; Delgado-Baquerizo et al., 2016). The importance of soil microbes in controlling of plant disease is well recognized and soil microbiome links to plant disease in many ways: stimulating production of plant growth hormones, competing with pathogens for nutrients, production of compounds (e.g., antibiotics) that inhibit pathogens or induce plant resistance to pathogens (Berendsen et al., 2012; Mendes et al., 2013). Because controlling plant pathogens by microbes is a sustainable and chemical-free approach, diseasesuppressive soils with beneficial microbes have been developed for biocontrol of plant diseases 
(Weller et al., 2002; Penton et al., 2014; Cha et al., 2016). The incidence or severity of disease is often lower in suppressive soils in comparison with that in surrounding soils (Cook and Baker, 1983).

Concerns about the impact of the soil microbiome on plant health has been examined in several studies (Sanguin et al., 2009; Qiu et al., 2012; Penton et al., 2014; Santhanam et al., 2015; Cha et al., 2016; Latz et al., 2016; Niu et al., 2016, 2017; Wang et al., 2017). Soil microbial community diversity, composition, function, and ecological relationship are all associated with plant soil borne disease outbreaks. Using a high-through put sequencing approach, Wang et al. (2017) found the microbial community was different between healthy and bacterial wilt-infected fields. Niu et al. (2016) also found that cropping regimes affected plant disease outbreaks by changing the soil microbial communities. Addition of organic fertilizers could inhibits plant diseases through alteration of soil microbial composition, diversity, and activity (Hunter et al., 2006). However, most studies have focused on the diversity and composition of the soil microbiome but ignored the microbial interactions.

Microbial interactions are a vital part of the soil microbiome (Zhang et al., 2014) and the interactions among different microbial groups are important for determining the ecosystem functioning/stability (Zhou et al., 2011), thus maybe a more integrated, complex network (many, stable interactions) is more difficult for a pathogen or other intruder to enter. Therefore, we hypothesized that complex network played some role in suppression of tobacco bacterial wilt disease. Many network approaches have been developed to reveal the interactions among microbial groups, e.g., equation-based network (Yeung et al., 2002), Bayesian network (Gerstung et al., 2009), relevance network (Horvath and Dong, 2008), CoNet network (Detti et al., 2011; Soffer et al., 2015) and random matrix theory (RMT)-based network (Deng et al., 2012). The threshold selection in the network construction is important, because the choice of thresholds have important effects on which OTUs are selected (Poudel et al., 2016). A RMTbased network can automatically identify the threshold for network construction (Zhou et al., 2010) using 16S rRNA gene sequencing data creating a molecular ecology network that is objective rather than subjective (Zhou et al., 2011; Deng et al., 2012).

The aim of the present study is to evaluate the relationship between soil microbiome and plant health and to reveal which microbes may play a role in inhibiting bacterial wilt disease. To reveal this relationship, we sampled soil samples in 15 tobacco farmlands (8-10 samples in each farmland) that were located in three different regions in central south of China. We investigated the soil microbial community using $16 \mathrm{~S}$ rRNA gene sequencing, constructed molecular ecological networks based on random matrix theory, analyzed network properties and inferred the key microorganisms in these networks. As a result, the present study offers an integrate insight into the relationship between soil microbial community and tobacco wilt disease.

\section{MATERIALS AND METHODS}

\section{Soil Samples and Wilt Infection Rate}

Soil samples were collected from 15 different tobacco fields located in different regions of Hunan province. Detailed location and other information are list in Supplementary Table S1. Eight to 10 samples were taken from each filed. Soil samples were collected using checkerboard sampling method as described in Niu et al. (2017). To be specific, each tobacco field was divided into 10 areas and upper layer soils $(0-20 \mathrm{~cm})$ at the central point of each area were sampled as one sample. The samples were collected on 28 th July, 2015 . Soil samples were stored at $-80^{\circ} \mathrm{C}$ before DNA extraction.

The wilt infection rate was calculated by the percentage of infected plants in each field. That is,

$$
\text { Infection rate }=\mathrm{Ni} / \mathrm{Nt} \times 100 \%,
$$

where $\mathrm{Ni}$ and $\mathrm{Nt}$ represents the number infected plants and the total number of plants in each field (about 250 plants), respectively.

\section{DNA Extraction, PCR Amplification, Sequencing and Data Processing}

DNA extraction, amplification of $16 \mathrm{~S}$ rRNA amplicons and sequencing were performed as described in our previous studies (Niu et al., 2016; Niu et al., 2017). Briefly, DNA were extracted using a MO BIO PowerSoil DNA Isolation Kit (MO BIO, San Diego) following the manufactures' manual. PCR amplification of 16S rRNA V4 region was performed on a Biosystems 2720 Thermal Cycler (ABI Inc., United States) with the primer pair 515F (5'-GTGCCAGCMGCCGCGGTAA-3') and 806R (5'-GGACTACHVGGGTWTCTAAT-3') primers together with Illumina adapter sequences and barcodes. The PCR reactions consisted of $0.2 \mu \mathrm{l}$ primers (10 nM each), $12.5 \mu \mathrm{l}$ Taq Master Mix (Vazyme, Piscataway, NJ, United States), $1.0 \mu$ I DNA template and $\mathrm{ddH} 2 \mathrm{O}$ to a final volume of $25 \mu \mathrm{l}$. PCR amplifications were performed as follows: desaturated at $95^{\circ} \mathrm{C}$ for $5 \mathrm{~min}$; followed by 30 cycles of $30 \mathrm{~s}$ at $95^{\circ} \mathrm{C}, 30 \mathrm{~s}$ at $55 / 59 / 62^{\circ} \mathrm{C}$ and $30 \mathrm{~s}$ at $72^{\circ} \mathrm{C}$ and a final extension step at $72^{\circ} \mathrm{C}$ for $10 \mathrm{~min}$. PCR products were recovered using gel extraction kit (OMEGA Bio-Tek Inc., Doraville, GA, United States) after subjecting the PCR mixture to agarose gel. Quality and quantity of recovered products were measured on Nanodrop Spectrophotometer (ND1000 Spectrophotometer, Nanodrop products, Wilmington, DE, United States). Sequencing libraries were constructed using $200 \mathrm{ng}$ of each purified PCR product and sequencing was performed on Illumina MiSeq machine (Illumina, San Diego, CA, United States) using the MiSeq 500 cycles kits. The Raw data were in fastq format. The $16 \mathrm{~S}$ rRNA gene sequences have been submitted to NCBI SRA database following the accession number of KR831285 - KR 855564.

Processing of sequencing data were performed on an inhouse galaxy pipeline developed in the lab of Dr. Zhou ${ }^{1}$. Firstly, reads were assigned to different samples according

\footnotetext{
${ }^{1}$ http://www.ou.edu/content/ieg/tools/data-analysis-pipeline.html
} 
to their barcode sequences and primer sequences were then removed. Subsequently, low quality reads (QC threshold $<20$ ) were removed using Btrim (Kong, 2014). Then, the forward and reverse reads with at least 10 bp overlap and less than 5\% mismatches were merged using Flash (Magoc and Salzberg, 2011), ambiguous bases (N) were removed from the merged sequences. Finally, reads with $97 \%$ similarity (Clustering threshold) were assigned to the same OTU, chimeras and singletons were removed and OTU table was generated using UPARSE (Edgar, 2013). To eliminate the influences caused by different sequencing depth, samples were normalized to 19 , 000 for further analysis. All downstream analyses including microbial community analyses and molecular ecology network construction were carried out using the normalized OTU table. Taxonomic assignment was carried out by blasting the sequences to RDP (Ribosomal Database Project) database with a 50\% minimal confidence.

\section{Random Matrix Theory Based Molecular Ecology Networks}

Molecular ecology networks (MENs) were constructed based on OTU abundance in each tobacco field, yielding a total of 15 networks. Only OTUs presented in 9 out of 10 (For site D, 8 out of 9 and for J, 7 out of 8) replicate samples were used for network construction. Because the criteria for selecting OTUs in network construction was different for site D and J, further analyses were carried out on the other 13 networks, and the results were similar to that obtained from 15 networks (data not shown). Networks were constructed as follows: paired valid missing values (a relative abundance of 0 ) was replaced by 0.01 , logarithm normalization of relative abundance was carried out to reduce the microbial community compositional bias (Faust and Raes, 2012), and similarity matrix was constructed based on Pearson Correlation Coefficient. Random matrix theory was used to choose the similarity threshold (St) automatically (Zhou et al., 2010, 2011; Deng et al., 2012). For network analysis, we characterized the global network properties and used greedy modularity optimization for module separation. All network analyses were performed on Institute for Environmental Genomics, The University of Oklahoma website ${ }^{2}$. Networks were visualized using Gephi software. Modules in each network were randomly colored, and modules with less than five nodes were colored gray.

\section{Statistical Analyses}

All calculations and statistical analyses were performed on $\mathrm{R}$ statistical platform (v 3.4.0). Community analyses were carried out based on OTU relative abundance in each tobacco planting field. Diversity indexes including Shannon diversity index, evenness and Chaol index were calculated using the 'vegan' package. Principle component analysis (PCA) was carried out using the 'vegan' package. Analysis of similarity (ANOSIM) and unweighted paired-group method with arithmetic means (UPGMA) based on Bray-Curtis distance matrix. To investigate any differences in microbial community in sites with different

${ }^{2}$ http://129.15.40.240/MENA/ wilt infection rate, PCA, UPGMA, and ANOSIM were performed using the average relative abundance of 8-10 replicates in each site. Partial Least Squares Path Modeling (PLSPM) was performed using 'amap', 'shape,' 'diagram,' and 'plspm' package. Pearson correlation analysis was used to calculate the correlation between two parameters. Significance level of difference between different groups was determined by one-way ANOVA (analysis of variance) followed by the Tukey's test. For unbalanced design, one-way ANOVA followed by Tukey's test was carried out using the General Linear Model function in Minitab 16.0 (Minitab inc.). A $p$-value of less than 0.05 was considered as significant.

\section{RESULTS}

\section{Soil Properties and Wilting Symptom}

The bacterial wilt infection rate ranged from 1.19 to $80.45 \%$ in all 15 sites (Supplementary Table S1). Six sites had an infection rate of less than 25\%, five sites had infection rates between 25 and $50 \%$ and four sites had an infection rate more than 50\%. Nine sites were the continual cropping of tobacco (Continues), while six sites were the rotation cropping of tobacco and other crops including lily, turnip and maize (Rotation). There is no statistically significant difference $(p>0.05$, ANOVA following Tukey's test) of infection rate among sample locations, nor any significant difference ( $p>0.05$, student $T$-test) between cultivation regimes (Rotation and Continual cropping).

Soil properties including $\mathrm{pH}$, water content, Potassium, Calcium and Ion contents were shown in Supplementary Table S2. Among them, $\mathrm{pH}$ (Pearson $=-0.606, p=0.017$ ) and Calcium (Pearson $=-0.591, p=0.020)$ showed significantly negative correlation with wilt infection rate (Supplementary Figure S1), and the remaining three parameters didn't show significant correlation with plant wilt symptom.

\section{Microbial Community}

A total of 8,366,296 reads were obtained from 147 samples, and 21,429 OTUs were identified from the reads. The rarefaction curves (Supplementary Figure S2) indicated that the sequencing depths were sufficient for downstream analysis. Fifteen sites had significantly different OTU numbers and Chaol indexes. Pearson correlation showed the mean observed OTU number and Chao1 index showed significant $(p<0.05)$ negative correlation with bacterial wilt infection rate (Figure 1A). In addition, all sites had similar Simpson diversity index (Figure 1B, from 0.975 to 0.995 ) and Pielou evenness (0.65-0.79). The Shannon diversity index differed among sites, and weakly correlated with wilt infection rate (Pearson index $=-0.485, p=0.067$ ).

Principle component analysis plot of all 147 samples (Figure 2A) showed that samples from individual sites grouped well with each other. The analysis of similarity (ANOSIM) ( $R=0.814, p=0.001)$ indicated that there were significant differences between groups (sites). To investigate the relationship between soil bacterial community composition and tobacco wilt disease, UPGMA tree (Figure 2B) and PCA (Figure 2C) were performed based on the average OTU relative abundance. UPGMA tree showed that samples with similar wilt infection 

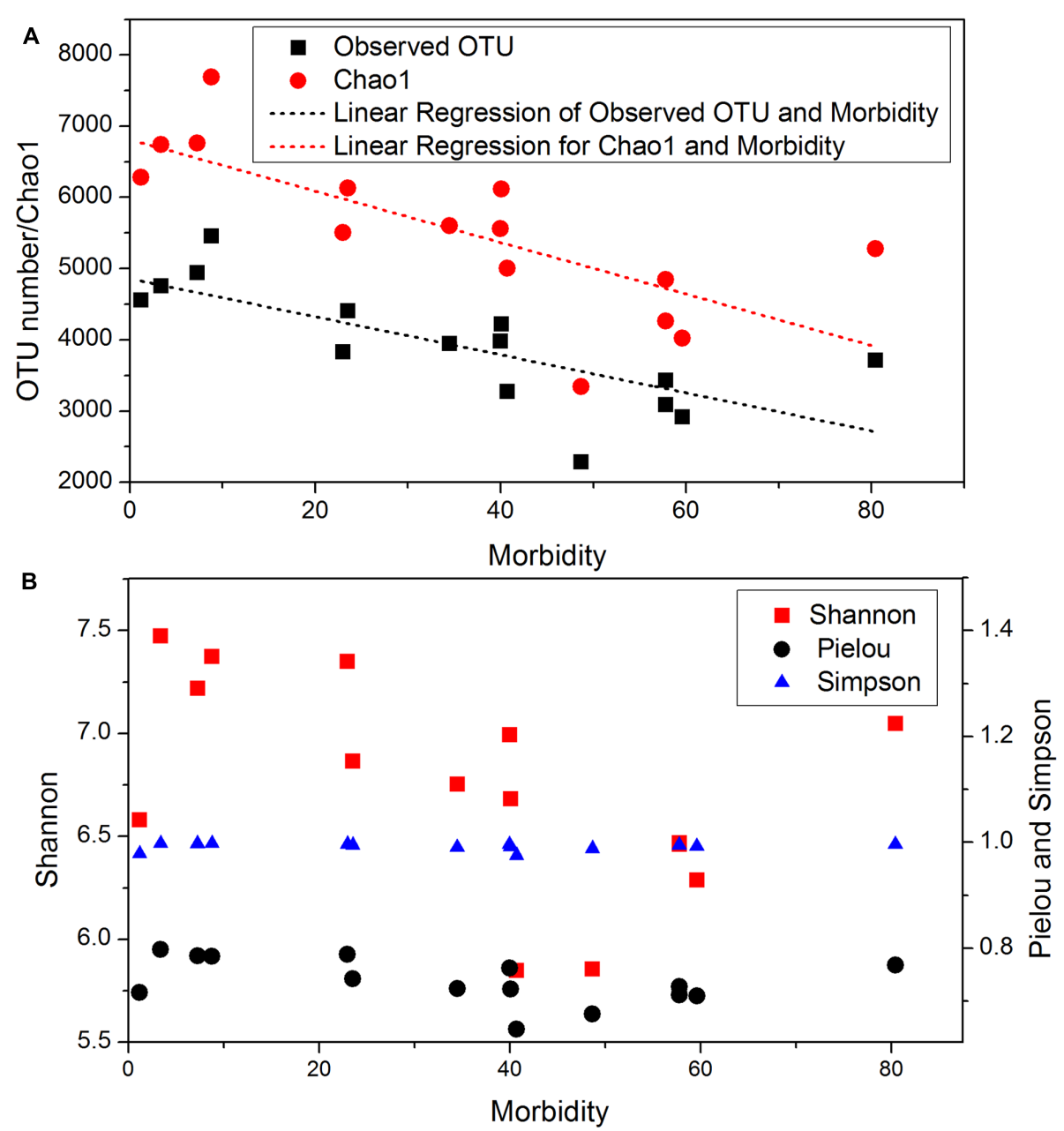

FIGURE 1 | Microbial community diversity in 15 sites with different bacterial wilt infection rate. (A) Observed OTU and Chao1; (B) Shannon, Pielou and Simpson indexes. Linear regressions were present for indexes that significantly ( $p$-value $<0.05)$ correlated with infection rate (Morbidity). Pearson correlation indexes and $p$-values are shown in Supplementary Table S5.

rates often clustered together. The two branches differed obviously in their wilt infection rate. The left branch was composed of five sites (I, J, C, L, M, and N) whose wilt infect rate was lower than $25 \%$ and we referred to these sites as 'healthy.' The 'infected' sites (D, E, A, B, K, G, O, F, and $\mathrm{H}$ ) are on the right branch. In addition, the 'infected' branch was further separated into the 'severely infected' [ $>50 \%$, with site D $(48.67 \%)$ as the exception] and the 'moderately infected' (25-50\%, with the site O $(80.48 \%)$ as the exception) branch. PCA plot of the bacterial communities in 15 sites showed similar results as UPGMA tree, 'healthy' and 'infected' groups separated along the first PCA axis. The 'healthy' sites are in the green circle, while the 'infected' sites are in the red circle. When all samples were classified into two groups (two branches on the UPMGA tree) according to the wilt infection rate, similarity analysis (ANOSIM) showed that the distances between groups was significantly larger than that within the groups $(R=0.7771$ and $p=0.001)$, suggesting that the two groups differed significantly in their microbial community composition.
All OTUs were classified into 931 genera and 39 phyla. The most abundant phylum in soils was the Proteobacteria which account for $33.49 \%$ of the bacterial community (Figure 3). Two phyla, Actinobacteria and candidate division WPS-2 significantly positive correlation with wilt infection rate (Supplementary Table S3). Ten phyla showed significantly negative correlation with wilt infection rate, which included Acidobacteria (Pearson $=-0.629, p=0.012)$, BRC1 (Pearson $=-0.762$, $p=0.001$ ), Crenarchaeota (Pearson $=-0.573, p=0.026$, Euryarchaeota $($ Pearson $=-0.612, p=0.015)$ Hydrogene-dentes (Pearson $=-0.559, p=0.030$ ), Ignavibacteriae (Pearson $=-0.607, p=0.016$ ), Latescibacteria (Pearson $=-0.651$, $p=0.009)$, Pacearchaeota (Pearson $=-0.737, p=0.002)$, Synergistetes (Pearson $=-0.677, p=0.006)$, and Woesearchaeota (Pearson $=-0.546, p=0.035)$. The most abundant genus in soils was Acidobacteria_GP16 (5.31\%), followed by Nitrososphaera (4.97\%), Sphingomonas (3.66\%), and Gemmatimonas (3.06\%), and the genus Acidobacteria_GP16 showed significantly negative correlation with tobacco wilt infection rate 

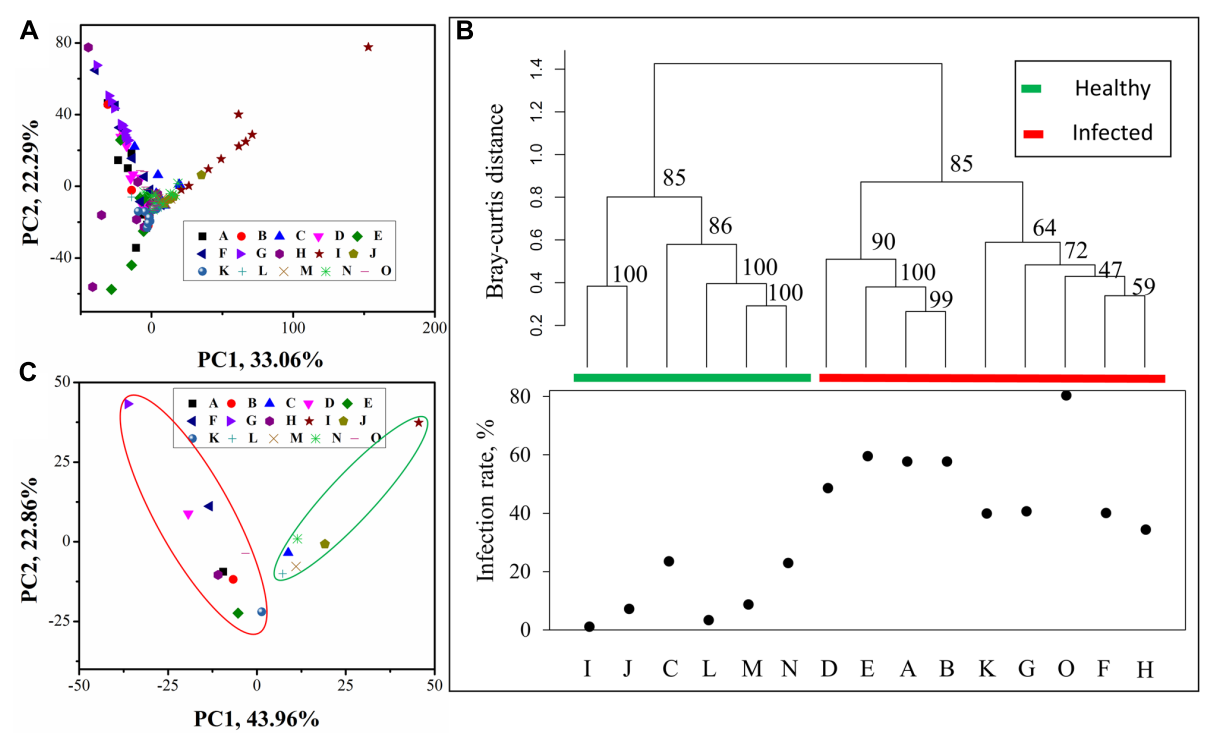

FIGURE 2 | Principle component analysis (PCA) and Unweighted paired group method with arithmetic mean (UPGMA) tree of microbial community composition. (A) PCA plot of all 147 samples grouped by sample sites; (B) UPGMA tree constructed from 'Bray-Curtis' distances between microbial community in each site, sites were clustered into two groups based on the community composition and as the two groups differed in the wilt infection rate, they were referred to 'healthy' (which had the infection rate lower than 25\%) and 'infected' group (which had the infection rate higher than 25\%), respectively. The numbers associated with the branches refer to bootstrap values (confidence limits) resulting from 100 replicate resamplings; (C) PCA plot of microbial community in sample sites. In (B,C), average relative abundance in each site was used for analysis.

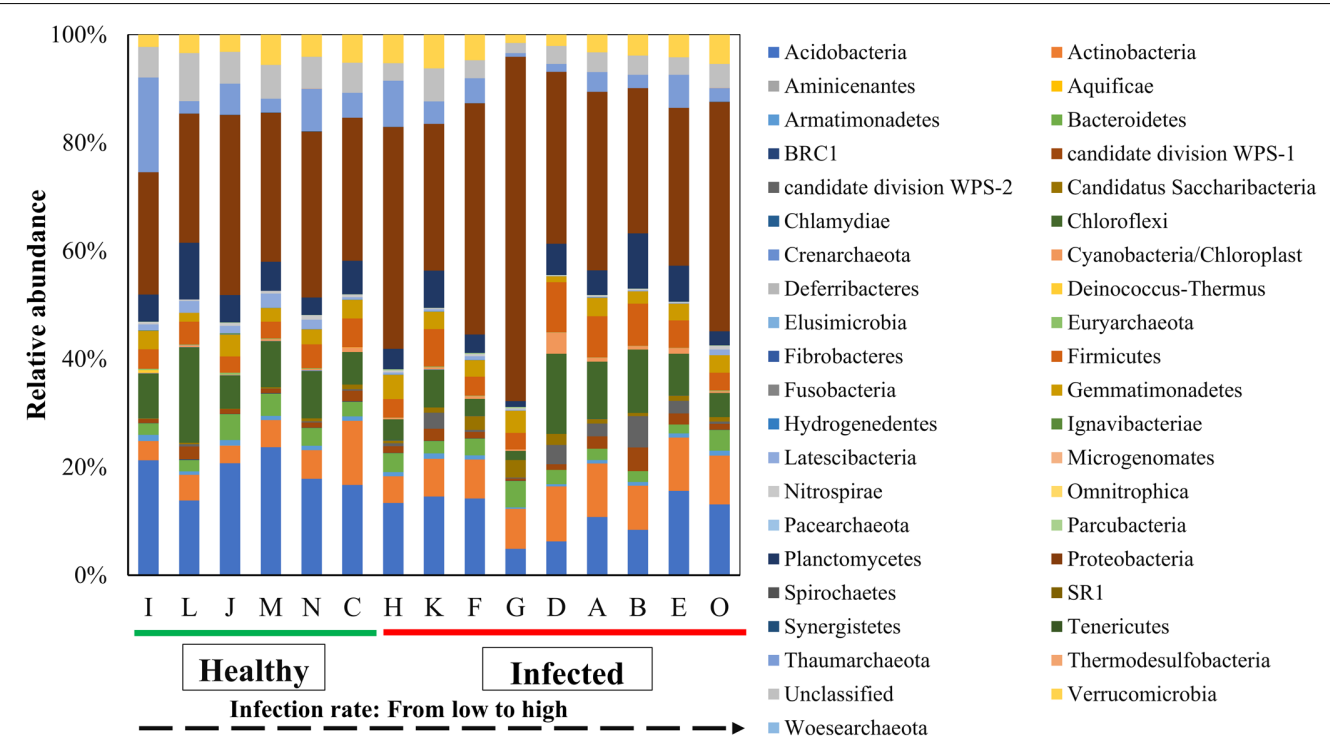

FIGURE 3 | Average relative abundance of bacterial phylum in soil samples. Results are means of 10 replicates. All sites can be grouped into two groups: 'Healthy' that has the infection rate of less than $25 \%$ and 'Infected' that has the infection rate more than $25 \%$.

(Pearson $=-0.641, p=0.010)$. As we focused on the wilt disease caused by Ralstonia, we investigated the relationship between relative abundance of the genus Ralstonia and the wilt disease. The genus Ralstonia had relative low abundance in soils $(0.002-0.21 \%)$ and showed significantly positive correlation with tobacco wilt morbidity (Pearson $=0.518$, $p=0.048)$.

\section{Molecular Ecology Networks}

To unravel the relationship between soil microbe-microbe interactions and tobacco bacterial wilt disease, we constructed a co-occurrence network for each field (Figure 4). The automatically generated similarity threshold $(s t)$ of the networks ranged from 0.89 to 0.94 (Supplementary Table S4) and powerlow $R$ square ranged from 0.82 to 0.93 (Supplementary Table $S 4$ ). 


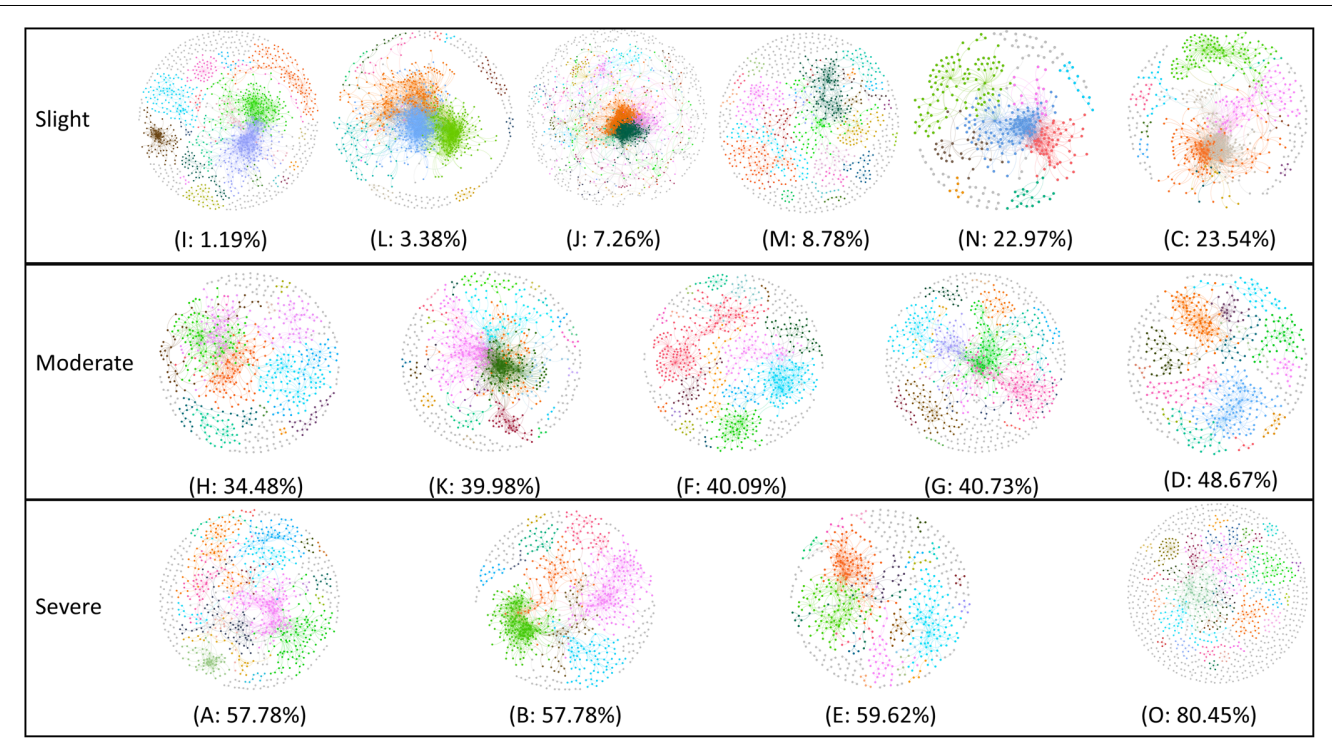

FIGURE 4 | Random matrix theory (RMT)-based molecular ecology networks in soils during Tobacco cultivation. Networks are present following the order of Tobacco bacterial wilt infection rate (from low to high). In the networks, nodes represent OTU and links represent significant correlation, modules are randomly colored and modules with less than five nodes are colored gray. Sites with relative low infection rate ( $<25 \%$, including I, L, J, M, N, and C) were referred to 'healthy' group, sites with relative high infection rate (>50\%, including $\mathrm{H}, \mathrm{A}, \mathrm{B}, \mathrm{E})$ were referred to 'severe' group and others that has the infection rate between 25 and $50 \%$ (K, F, G, O and D) were referred to 'moderate' group.

The st were chosen using the RMT-based theory to obtain an adjacency matrix, which represents the strength of the connection between each pair of OTUs. Similar St indicated that the minimum strength of the connection in all networks was similar. The $R$ square values were near to 1 indicating that the goodness of fit was proper. The network properties differed obviously among sites (Supplementary Table S4), for example, the nodes ranged from 398 to 1436, the links ranged from 1139 to 12580 , the average degree ranged from 2.35 to 17.90 and the modules ranged from 30 to 208. The sites that with healthy plants tended to have more complex networks based on the average degree and number of links that showed significant negative correlation with wilt incidence. The network complexity can be described by the number of links and average connectivity (average $K$ ) with more links and higher average $K$ indicative of a more complex network (Deng et al., 2012). Furthermore, modularity is an important concept in networks and microbial populations in the same module have similar ecological niches (Deng et al., 2012). Pearson correlation analysis indicated that average $K$ (Pearson $=-0.656, p=0.008)$, number of links (Pearson $=-0.684, p=0.005$ ) and number of modules (Pearson $=-0.543, p=0.037$ ) showed a strong negative correlation with Tobacco bacterial wilt infection (Table 1), meaning sites with more complex and more modular networks have lower wilt infection rate.

\section{Network Key Taxa}

To reveal the key microbes in the networks, we looked into the nodes with max degree, max betweenness, max stress centrality, and $\max$ eigenvector centrality (Table 2). Nodes with max degree are the nodes that have the most interactions with other nodes (Chaffron et al., 2010; Deng et al., 2012). Betweenness and stress centrality are similar to each other, nodes with highest betweenness (or stress centrality) can serve as brokers (Guimera et al., 2007; Deng et al., 2012). The highest eigenvector centrality means the nodes have the highest degree when they connected to other central nodes (Bonacich, 1987). These nodes are important in maintaining the microbe-microbe interactions in the networks. Table 2 shows the key OTUs and their taxa in all networks. Members in phylum Proteobacteria and Acidobacteria are the main key taxa 'healthy' networks (e.g., members in phylum Acidobacteria acted as key taxa in I, J, and $\mathrm{C}$, while members in phylum Proteobacteria in I, M, N, and C). Members in phylum Actinobacteria (e.g., genus Gaiella) and Firmicutes (e.g., genus Bacillus) are also important taxa in networks from fields with healthy plants. Key taxa varied among sites that showed moderate wilt infection, and included members in phylum Gemmatimonadetes (e.g., genus Gemmatimonas), Firmicutes (e.g., genus Lysinibacillus), Acidobacteria (e.g., genus Gp16), Nitrospirae (e.g., genus Nitrospira) and else. In soils with plants severely infected by bacterial wilt, the network maintaining taxa also varied.

\section{DISCUSSION}

Multiple soil factors are responsible for bacterial wilt infection as the soil microbiome plays an important part of tobacco wilt severity. Because plants often lack genetic resistance to most necrotrophic pathogens, soil antagonistic microbes would protect the plants against pathogens (Cha et al., 2016). Pathogens of the genus Ralstonia are well-known for their 
TABLE 1 | Pearson correlation between wilt infection rate and microbial community which are related to microbial community diversity, composition and network properties.

\begin{tabular}{|c|c|c|c|c|c|c|c|c|c|c|c|}
\hline & & \multicolumn{4}{|c|}{ Diversity } & \multicolumn{2}{|c|}{ Composition } & \multicolumn{4}{|c|}{ Network } \\
\hline & & Diversity & Evenness & Observed OTU & Chao1 & PC1 & PC2 & Nodes & Links & Avg.K & Modules \\
\hline \multirow[t]{2}{*}{ Wilt } & Pearson & -0.485 & -0.402 & -0.751 & -0.747 & -0.639 & -0.252 & -0.317 & -0.684 & -0.656 & -0.543 \\
\hline & $p$-value & 0.067 & 0.137 & 0.001 & 0.001 & 0.01 & 0.365 & 0.250 & 0.005 & 0.008 & 0.037 \\
\hline
\end{tabular}

Avg.K, average degree.

TABLE 2 | Important microbes in networks.

\begin{tabular}{|c|c|c|c|c|}
\hline & Nodes with max degree & $\begin{array}{l}\text { Nodes with max } \\
\text { betweenness }\end{array}$ & $\begin{array}{c}\text { Nodes with max stress } \\
\text { centrality }\end{array}$ & $\begin{array}{c}\text { Nodes with max eigenvector } \\
\text { centrality }\end{array}$ \\
\hline । & $\begin{array}{c}\text { OTU_24040; Acidobacteria, } \\
\text { Gp7 }\end{array}$ & OTU_7856, Acidobacteria, Gp6 & $\begin{array}{c}\text { OTU_5231, Proteobacteria, } \\
\text { Thiohalobacter }\end{array}$ & $\begin{array}{c}\text { OTU_20616, Acidobacteria, } \\
\text { Gp6 }\end{array}$ \\
\hline$L$ & $\begin{array}{l}\text { OTU_747, Chloroflexi, } \\
\text { Thermomarinilinea }\end{array}$ & OTU_359, Acidobacteria, Gp25 & $\begin{array}{l}\text { OTU_747, Chloroflexi, } \\
\text { Thermomarinilinea }\end{array}$ & OTU_340, Acidobacteria, Gp6 \\
\hline$J$ & OTU_214, Acidobacteria, Gp4 & OTU_909, Firmicutes, Bacillus & $\begin{array}{l}\text { OTU_909, Firmicutes, } \\
\text { Bacillus }\end{array}$ & OTU_214, Acidobacteria, Gp4 \\
\hline M & $\begin{array}{c}\text { OTU_21670, Proteobacteria, } \\
\text { Variovorax }\end{array}$ & $\begin{array}{c}\text { OTU_113, Actinobacteria, } \\
\text { Gaiella }\end{array}$ & $\begin{array}{c}\text { OTU_113, Actinobacteria, } \\
\text { Gaiella }\end{array}$ & OTU_478, Unclassified, \\
\hline $\mathrm{N}$ & $\begin{array}{c}\text { OTU_193, Proteobacteria, } \\
\text { Hyphomicrobium }\end{array}$ & $\begin{array}{c}\text { OTU_193 Proteobacteria, } \\
\text { Hyphomicrobium }\end{array}$ & $\begin{array}{c}\text { OTU_193, Proteobacteria, } \\
\text { Hyphomicrobium }\end{array}$ & $\begin{array}{c}\text { OTU_749, Proteobacteria, } \\
\text { Azoarcus }\end{array}$ \\
\hline C & $\begin{array}{c}\text { OTU_24084, Proteobacteria, } \\
\text { Curvibacter }\end{array}$ & $\begin{array}{c}\text { OTU_720, Proteobacteria, } \\
\text { Moraxellaceae(F) }\end{array}$ & $\begin{array}{c}\text { OTU_720, Proteobacteria, } \\
\text { Moraxellaceae(F) }\end{array}$ & OTU_214, Acidobacteria, Gp4 \\
\hline K & $\begin{array}{c}\text { OTU_18948, Planctomycetes, } \\
\text { Singulisphaera }\end{array}$ & $\begin{array}{c}\text { OTU_18948, Planctomycetes, } \\
\text { Singulisphaera }\end{array}$ & $\begin{array}{c}\text { OTU_17, Firmicutes, } \\
\text { Unclassified }\end{array}$ & $\begin{array}{c}\text { OTU_21, Planctomycetes, } \\
\text { Thermogutta }\end{array}$ \\
\hline $\mathrm{F}$ & $\begin{array}{l}\text { OTU_12483, } \\
\text { Gemmatimonadetes, } \\
\text { Gemmatimonas }\end{array}$ & $\begin{array}{l}\text { OTU_204, } \\
\text { Gemmatimonadetes, } \\
\text { Gemmatimonas }\end{array}$ & $\begin{array}{l}\text { OTU_204, } \\
\text { Gemmatimonadetes, } \\
\text { Gemmatimonas }\end{array}$ & $\begin{array}{c}\text { OTU_43, Proteobacteria, } \\
\text { Denitratisoma }\end{array}$ \\
\hline G & $\begin{array}{l}\text { OTU_123, Verrucomicrobia, } \\
\text { Spartobacteria_genera_incertae_sedis } \\
\text { OTU_36, Nitrospirae, Nitrospira }\end{array}$ & $\begin{array}{c}\text { OTU_13176, Acidobacteria, } \\
\text { Gp16 }\end{array}$ & $\begin{array}{c}\text { OTU_13176, } \\
\text { Acidobacteria, Gp16 }\end{array}$ & OTU_36, Nitrospirae, Nitrospira \\
\hline $\mathrm{H}$ & OTU_229, Acidobacteria, Gp3 & $\begin{array}{l}\text { OTU_289, Firmicutes, } \\
\text { Lysinibacillus }\end{array}$ & $\begin{array}{c}\text { OTU_71, Chloroflexi, } \\
\text { Unclassified }\end{array}$ & $\begin{array}{c}\text { OTU_91, Bacteroidetes, } \\
\text { Chitinophaga }\end{array}$ \\
\hline $\mathrm{D}$ & $\begin{array}{c}\text { OTU_2693, Proteobacteria, } \\
\text { Ectothiorhodosinus }\end{array}$ & $\begin{array}{l}\text { OTU_1565, Chloroflexi, } \\
\text { Ktedonobacterales(O) }\end{array}$ & $\begin{array}{l}\text { OTU_1565, Chloroflexi, } \\
\text { Ktedonobacterales(O) }\end{array}$ & $\begin{array}{c}\text { OTU_1399, Actinobacteria, } \\
\text { Conexibacter }\end{array}$ \\
\hline A & $\begin{array}{l}\text { OTU_22415, Proteobacteria, } \\
\quad \text { Xanthomonadaceae (F) }\end{array}$ & $\begin{array}{l}\text { OTU_5454 Armatimonadetes, } \\
\text { Armatimonadetes_gp4 }\end{array}$ & $\begin{array}{c}\text { OTU_5454, } \\
\text { Armatimonadetes, } \\
\text { Armatimonadetes_gp4 }\end{array}$ & $\begin{array}{l}\text { OTU_4756, Chloroflexi, } \\
\text { Ktedonobacter }\end{array}$ \\
\hline$B$ & $\begin{array}{c}\text { OTU_183, Proteobacteria, } \\
\text { Unclassified }\end{array}$ & $\begin{array}{c}\text { OTU_24237, Planctomycetes, } \\
\text { Rubinisphaera }\end{array}$ & $\begin{array}{l}\text { OTU_24237, } \\
\text { Planctomycetes, } \\
\text { Rubinisphaera }\end{array}$ & $\begin{array}{l}\text { OTU_82, Chloroflexi, } \\
\text { Ktedonobacter }\end{array}$ \\
\hline$E$ & OTU_2035, Acidobacteria, Gp6 & $\begin{array}{c}\text { OTU_9618, Proteobacteria, } \\
\text { Unclassified }\end{array}$ & $\begin{array}{c}\text { OTU_9618, Proteobacteria, } \\
\text { Unclassified }\end{array}$ & $\begin{array}{c}\text { OTU_6, Verrucomicrobia, } \\
\text { Spartobacteria_genera } \\
\text { _incertae_sedis }\end{array}$ \\
\hline $\mathrm{O}$ & OTU_45 Acidobacteria, Gp6 & $\begin{array}{c}\text { OTU_1301, Proteobacteria, } \\
\text { Neisseriaceae }(F)\end{array}$ & $\begin{array}{c}\text { OTU_1301, Proteobacteria, } \\
\text { Neisseriaceae (F) }\end{array}$ & OTU_45, Acidobacteria, Gp6 \\
\hline
\end{tabular}

The classification levels are indicated in the brackets. O, oreder; F, family. If not specified, the taxa levels shown are phylum and genus.

ability to cause wilt symptoms in members of the Solanaceous family (Hayward, 1991; Chen et al., 2013). In this study, we focused on the relationship between the soil microbiome and tobacco Ralstonia wilt infection and found that wilt infection was closely related to the diversity, composition, and inferred microbe-microbe interactions of soil microbial communities.

Tobacco wilt infection was associated with soil microbial community diversity, as indicated by observed OTU number and
Chao1 index. Bacterial OTUs were more diverse in fields with healthy plants than in fields with infection. Even though the correlation between the Shannon index and wilt infection was not strong, our results showed that the microbial community diversity had a positive correlation with wilt infection rate. This is similar to a recent report that the microbial community diversity was higher in soils with healthy plants than in soils with plants infected by bacterial wilt (Wang et al., 2017). It has also been reported that the rhizosphere soil microbial community 


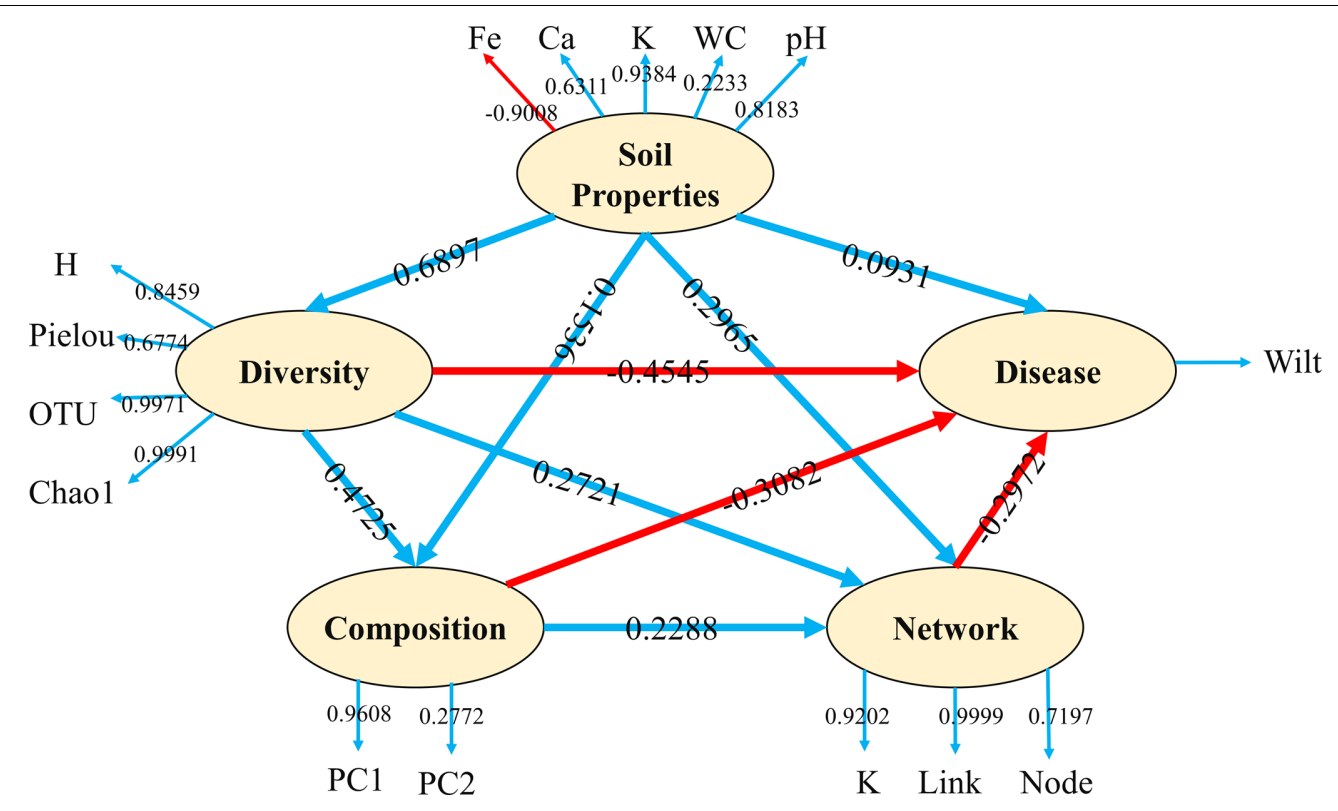

FIGURE 5 | Partial Least Squares Path Modeling (PLSPM). Blue lines represent positive effects and red lines represent negative effect. Numbers on the lines in the PLSPM model are the 'total effects' values. Numbers on the lines out of the PLSPM model are the 'weight' contributions. WC, water content; H, Shannon diversity index; $\mathrm{K}$, average degree.

diversity is positively correlated with plant health (Bulluck and Ristaino, 2002; Luan et al., 2015). The results suggest that a highly diverse soil microbiome (greater numbers of microbial species) would decrease the chances of bacterial wilt outbreak. Many studies have also found that a diverse microbial community was often less prone to pathogens invasion than a simpler microbial community (Case, 1990; Fargione and Tilman, 2005; van Elsas et al., 2012).

Interestingly, all filed sites clustered into 'healthy' and 'infected groups, in the UPGMA tree or the first PCA axis and that this clustering was not related to either the geographic locations or cultivation regimes. The 'healthy' $(0-25 \%)$ branch of soil samples was from Huahenger (C), Pailiao (I and J) or Fenghuang ( $\mathrm{L}, \mathrm{M}$, and $\mathrm{N})$, and 'infected' branch $(>25 \%)$ was based on rotations (D and $\mathrm{E}$ ) or continuous (A and $\mathrm{B}$ ) cultivation regimes. These results suggested that soil microbial community composition could have played a role in preventing tobacco from wilt pathogens infections. This may be because certain populations of beneficial soil microbes were enhanced in sites with low incidence of bacterial wilt. For example, Bulluck and Ristaino (2002) found that the increase of beneficial soil microbes significantly reduced the outbreak chance of southern blight. In this context, soil microbial communities with abundant biocontrol agents (BCAs) or pathogenic antagonists may be able to control the incidence of soil borne bacterial diseases. Even though not all BCAs or pathogenic antagonists are known and some BCAs or pathogenic antagonistic members could become plant pathogens under specific circumstances, we can propose that soils with a similar microbial community structure of the 'healthy' branch are likely to suppress the outbreak of tobacco wilt disease.
To further reveal which soil microbes may be important for inhibiting wilt disease outbreaks, we attempted to identify 'inferred' key microbial groups in molecular ecological networks. In networks, nodes with max connectivity (degree), max betweenness, stress centrality and eigenvector centrality play important roles in maintaining network structure (Olesen et al., 2007; Zhou et al., 2011; Faust and Raes, 2012). This is because (i) nodes with max degree have the most edges in the network, (ii) nodes with max betweenness and stress centrality can serve as brokers in the network and (iii) nodes with max eigenvector centrality have the highest connections with other central nodes. Furthermore, it is also supposed that the taxa of these nodes play important roles in maintaining ecosystem stability (Olesen et al., 2007; Lu et al., 2013). In this study, these key taxa were relatively consistent in fields with healthy plants (branch 1 on the UPGMA tree), suggesting that these taxa might be important in the suppression of bacteria wilt disease. In fields with healthy plants, taxa considered important for maintaining ecosystem stability were mainly Bacillus in the phylum Firmicutes, several members in the phylum Actinobacteria and a few other organisms. These taxa are often considered as plant-beneficial microbes. Members of the genus Bacillus are well-known as BCAs (Emmert and Handelsman, 1999; Schisler et al., 2004; Ongena and Jacques, 2008). Due to their ability to secrete antibiotics or antimicrobial proteins (Ahimou et al., 2000; Moyne et al., 2004), they showed a wide range of biological effects on plant pathogens and have been applied to control bacterial diseases of alfalfa (Handelsman et al., 1990), tobacco (Fravel et al., 1977) and cucumber (Cao et al., 2011). Many members of Actinobacteria have also shown the ability to control plant bacterial diseases (Gomes et al., 2000; Doumbou et al., 2001; Kinkel et al., 2012; 
Palaniyandi et al., 2013). Actinobacteria are well-known antagonistic microbes of many pathogens, as they can (i) produce a diverse range of antibiotics (Liu et al., 2012), (ii) secret cell-wall degrading enzymes (Chater et al., 2010), and/or (iii) induce host (plant) resistance (Conn et al., 2008). In the wilt disease infected fields (branch 2), key taxa for maintaining ecosystem stability varied. As sites with higher wilt infection rate formed simple networks (fewer edges and lower average degree) with fewer modules, it is possible that some key bacteria in disease-causing soils inhibited the formation of complex, modular networks that were characteristic of suppressive soils. In this context, we proposed that they could possibly promote the formation of complex, modular microbial community networks, thus making soils wiltsuppressive when plant-beneficial taxa played important roles in maintaining the ecosystem stability. Taking the microbial community composition and the important taxa together, the results indicate that when the soil microbial community composition is similar to that of the 'healthy' group with plantbeneficial microbes, the soil ecosystem may remain stable and disease-suppressive.

We proposed that complex, modular microbial community networks might make soils wilt-suppressive, however, since the soil ecology is very complex, with the flow of energy, matter, and information not well understood a majority of the soil microbes still unculturable, most of the microbemicrobe interactions remain unclear. More research is still needed to reveal the relationship between below-ground ecology networks and above-ground plant health. Previous studies have indicated that networks could be altered by environmental properties, such as $\mathrm{pH}$ (Tylianakis et al., 2007; Barberán et al., 2012; Shi et al., 2016). This was consistent with the present study which showed a significant correlation between soil $\mathrm{pH}$ and network complexity (Supplementary Table S5). We also observed that the bacterial community diversity showed significant positive correlation with the network complexity, which is, however, in contrast with the finding in rhizosphere networks (Shi et al., 2016) whose complexity was accompanied by decreased bacterial diversity. This might be because the rhizosphere and the surrounding bulk soils have very different bacterial communities (Kourtev et al., 2002; Berg and Smalla, 2009; Shi et al., 2015) and ecological networks were more complicated in rhizosphere than in surrounding bulk soils.

The present study showed a range of correlations between plant wilt infection and soil microbial properties. We conducted PLSPM analyses (Figure 5) to profile the complex relationship between soil microbial community properties and plant health. Soil properties had strong effect on microbial diversity and weak direct effect on microbial composition, microbial networks and disease infections, however, soil properties can also affect plant disease infection indirectly through soil microbial diversity, composition and network interactions. Among soil microbial properties, microbe diversity showed the strongest effects (negatively, -0.4545) on plant disease infection, followed by microbial community composition (negatively,
-0.3082 and molecular ecology network complexity (negatively, $-0.2972)$.

In summary, the present study offered an integrate view of the relationship between soil bacterial community and plant health. We found that (i) soil microbial diversity had a strong effect on plant disease level, with diversity and rate of wilt disease showing an inverse relationship; and (ii) soils with similar microbial community composition have similar disease infection rate. When the soil had similar composition with 'healthy' group, the soil might tend to be disease-suppressive. According to the inferred molecular ecology networks, we proposed that a more complex network might be beneficial for tobacco wilt suppression. In addition, some potential plant-beneficial microbial groups could act as network key taxa, thus reducing the chance of plant soil-borne pathogen invasion. However, as the soil microbial ecology network are extremely complex, more work, particularly the experimental work, is still needed to test the proposal. We concluded that microbial community in disease-suppressive soils may be consisted with high diversity, consistent composition with plantbeneficial microbes as the important component and complex network.

\section{AUTHOR CONTRIBUTIONS}

HYa, YL, HL, and HYi conceived and designed the experiments. $\mathrm{YL}, \mathrm{HL}, \mathrm{XL}$, and HYi contributed reagents and materials. JH, JL, and YG performed the experiments. DM, YG, and YX analyzed the data. DM, JH, YX, and HYi wrote and revised the paper.

\section{FUNDING}

This research was supported by the Key Project of Science and Technology of Hunan Branch of China National Tobacco Corporation (16-19Aa02), the Changsha Branch of Hunan Tobacco Corporation (20140310012), the Chinese Postdoctoral Funding Plan and the Central South University Postdoctoral Research Funding for DM (201699).

\section{ACKNOWLEDGMENTS}

The authors would like to thank the Hunan Branch of China National Tobacco Corporation and Postdoctoral fellowship at CSU for funding the work. We would also like to thank Dr. James W. Voordeckers for language editing.

\section{SUPPLEMENTARY MATERIAL}

The Supplementary Material for this article can be found online at: https://www.frontiersin.org/articles/10.3389/fmicb. 2017.02179/full\#supplementary-material 


\section{REFERENCES}

Ahimou, F., Jacques, P., and Deleu, M. (2000). Surfactin and iturin A effects on Bacillus subtilis surface hydrophobicity. Enzyme Microb. Technol. 27, 749-754. doi: 10.1016/S0141-0229(00)00295-7

Barberán, A., Bates, S. T., Casamayor, E. O., and Fierer, N. (2012). Using network analysis to explore co-occurrence patterns in soil microbial communities. ISME J. 6, 343-351. doi: 10.1038/ismej.2011.119

Berendsen, R. L., Pieterse, C. M., and Bakker, P. A. (2012). The rhizosphere microbiome and plant health. Trends Plant Sci. 17, 478-486. doi: 10.1016/j. tplants.2012.04.001

Berg, G., and Smalla, K. (2009). Plant species and soil type cooperatively shape the structure and function of microbial communities in the rhizosphere. FEMS Microbiol. Ecol. 68, 1-13. doi: 10.1111/j.1574-6941.2009.00654.x

Bonacich, P. (1987). Power and centrality: a family of measures. Am. J. Soc. 92, 1170-1182. doi: $10.1086 / 228631$

Bulluck, L. III, and Ristaino, J. (2002). Effect of synthetic and organic soil fertility amendments on southern blight, soil microbial communities, and yield of processing tomatoes. Phytopathology 92, 181-189. doi: 10.1094/PHYTO.2002. 92.2.181

Cao, Y., Zhang, Z., Ling, N., Yuan, Y., Zheng, X., Shen, B., et al. (2011). Bacillus subtilis SQR 9 can control Fusarium wilt in cucumber by colonizing plant roots. Biol. Fertil. Soils 47, 495-506. doi: 10.1007/s00374-011-0556-2

Case, T. J. (1990). Invasion resistance arises in strongly interacting species-rich model competition communities. Proc. Natl. Acad. Sci. U.S.A. 87, 9610-9614. doi: 10.1073/pnas.87.24.9610

Cha, J. Y., Han, S., Hong, H. J., Cho, H., Kim, D., Kwon, Y., et al. (2016). Microbial and biochemical basis of a Fusarium wilt-suppressive soil. ISME J. 10, 119-129. doi: 10.1038/ismej.2015.95

Chaffron, S., Rehrauer, H., Pernthaler, J., and von Mering, C. (2010). A global network of coexisting microbes from environmental and whole-genome sequence data. Genome Res. 20, 947-959. doi: 10.1101/gr.104521.109

Chater, K. F., Biró, S., Lee, K. J., Palmer, T., and Schrempf, H. (2010). The complex extracellular biology of Streptomyces. FEMS Microbiol. Rev. 34, 171-198. doi: 10.1111/j.1574-6976.2009.00206.x

Chen, Y., Yan, F., Chai, Y., Liu, H., Kolter, R., Losick, R., et al. (2013). Biocontrol of tomato wilt disease by Bacillus subtilis isolates from natural environments depends on conserved genes mediating biofilm formation. Environ. Microbiol. 15, 848-864. doi: 10.1111/j.1462-2920.2012.02860.x

Conn, V., Walker, A., and Franco, C. (2008). Endophytic actinobacteria induce defense pathways in Arabidopsis thaliana. Mol. Plant Microbe Interact. 21, 208-218. doi: 10.1094/MPMI-21-2-0208

Cook, R. J., and Baker, K. F. (1983). The Nature and Practice of Biological Control of Plant Pathogens. St. Paul, MN: American Phytopathological Society.

Delgado-Baquerizo, M., Maestre, F. T., Reich, P. B., Jeffries, T. C., Gaitan, J. J., Encinar, D., et al. (2016). Microbial diversity drives multifunctionality in terrestrial ecosystems. Nat. Commun. 7:10541. doi: 10.1038/ncomms10541

Deng, Y., Jiang, Y.-H., Yang, Y., He, Z., Luo, F., and Zhou, J. (2012). Molecular ecological network analyses. BMC Bioinformatics 13:113. doi: 10.1186/14712105-13-113

Detti, A., Blefari Melazzi, N., Salsano, S., and Pomposini, M. (2011). "CONET: a content centric inter-networking architecture," in Proceedings of the ACM SIGCOMM Workshop on Information-Centric Networking (Hong Kong: ACM), 50-55.

Doumbou, C. L., Hamby Salove, M., Crawford, D. L., and Beaulieu, C. (2001). Actinomycetes, promising tools to control plant diseases and to promote plant growth. Phytoprotection 82, 85-102. doi: 10.7202/706219ar

Edgar, R. (2013). UPARSE: highly accurate OTU sequences from microbial amplicon reads. Nat. Methods 10, 996-998. doi: 10.1038/nmeth.2604

Emmert, E. A., and Handelsman, J. (1999). Biocontrol of plant disease: a (Gram-) positive perspective. FEMS Microbiol. Lett. 171, 1-9. doi: 10.1111/j.1574-6968. 1999.tb13405.x

Fargione, J. E., and Tilman, D. (2005). Diversity decreases invasion via both sampling and complementarity effects. Ecol. Lett. 8, 604-611. doi: 10.1111/j. 1461-0248.2005.00753.x

Faust, K., and Raes, J. (2012). Microbial interactions: from networks to models. Nat. Rev. Microbiol. 10, 538-550. doi: 10.1038/nrmicro2832
Fravel, D. R., Spurr, H., and Harvey, J. (1977). Biocontrol of tobacco brownspot disease by Bacillus cereus subsp. mycoides in a controlled environment. Phytopathology 67, 930-932. doi: 10.1094/Phyto-67-930

Gerstung, M., Baudis, M., Moch, H., and Beerenwinkel, N. (2009). Quantifying cancer progression with conjunctive Bayesian networks. Bioinformatics 25, 2809-2815. doi: 10.1093/bioinformatics/btp505

Gomes, R., Semedo, L., Soares, R., Alviano, C., Linhares, L., and Coelho, R. (2000). Chitinolytic activity of actinomycetes from a cerrado soil and their potential in biocontrol. Lett. Appl. Microbiol. 30, 146-150. doi: 10.1046/j.1472-765x.2000. 00687.x

Guimera, R., Sales-Pardo, M., and Amaral, L. A. (2007). Classes of complex networks defined by role-to-role connectivity profiles. Nat. Phys. 3, 63-69. doi: 10.1038/nphys489

Handelsman, J., Raffel, S., Mester, E. H., Wunderlich, L., and Grau, C. R. (1990). Biological control of damping-off of alfalfa seedlings with Bacillus cereus UW85. Appl. Environ. Microbiol. 56, 713-718.

Hayward, A. (1991). Biology and epidemiology of bacterial wilt caused by Pseudomonas solanacearum. Annu. Rev. Phytopathol. 29, 65-87. doi: 10.1146/ annurev.py.29.090191.000433

Horvath, S., and Dong, J. (2008). Geometric interpretation of gene coexpression network analysis. PLOS Comput. Biol. 4:e1000117. doi: 10.1371/journal.pcbi. 1000117

Hunter, P. J., Petch, G. M., Calvo-Bado, L. A., Pettitt, T. R., Parsons, N. R., Morgan, J. A. W., et al. (2006). Differences in microbial activity and microbial populations of peat associated with suppression of damping-off disease caused by Pythium sylvaticum. Appl. Environ. Microbiol. 72, 6452-6460. doi: 10.1128/ AEM.00313-06

Janvier, C., Villeneuve, F., Alabouvette, C., Edel-Hermann, V., Mateille, T., and Steinberg, C. (2007). Soil health through soil disease suppression: which strategy from descriptors to indicators? Soil Biol. Biochem. 39, 1-23. doi: 10.1016/j. soilbio.2006.07.001

Kinkel, L. L., Schlatter, D. C., Bakker, M. G., and Arenz, B. E. (2012). Streptomyces competition and co-evolution in relation to plant disease suppression. Res. Microbiol. 163, 490-499. doi: 10.1016/j.resmic.2012.07.005

Kong, Y. (2014). Btrim: a fast, lightweight adapter and quality trimming program for next-generation sequencing technologies. Genomics 98, 152-153. doi: 10.1016/j.ygeno.2011.05.009

Kourtev, P. S., Ehrenfeld, J. G., and Häggblom, M. (2002). Exotic plant species alter the microbial community structure and function in the soil. Ecology 83, 3152-3166. doi: 10.1890/0012-9658(2002)083[3152:EPSATM]2.0.CO;2

Latz, E., Eisenhauer, N., Rall, B. C., Scheu, S., and Jousset, A. (2016). Unravelling linkages between plant community composition and the pathogen-suppressive potential of soils. Sci. Rep. 6:23584. doi: 10.1038/srep23584

Liu, X., Bolla, K., Ashforth, E. J., Zhuo, Y., Gao, H., Huang, P., et al. (2012). Systematics-guided bioprospecting for bioactive microbial natural products. Antonie Van Leeuwenhoek 101, 55-66. doi: 10.1007/s10482-011-9671-1

Lu, L., Yin, S., Liu, X., Zhang, W., Gu, T., Shen, Q., et al. (2013). Fungal networks in yield-invigorating and-debilitating soils induced by prolonged potato monoculture. Soil Biol. Biochem. 65, 186-194. doi: 10.1016/j.soilbio. 2013.05.025

Luan, F., Zhang, L., Lou, Y., Wang, L., Liu, Y., and Zhang, H. (2015). Analysis of microbial diversity and niche in rhizosphere soil of healthy and diseased cotton at the flowering stage in southern Xinjiang. Genet. Mol. Res. 14, 1602-1611. doi: 10.4238/2015.March.6.7

Magoc, T., and Salzberg, S. L. (2011). FLASH: fast length adjustment of short reads to improve genome assemblies. Bioinformatics 27, 2957-2963. doi: 10.1093/ bioinformatics/btr507

Mendes, R., Garbeva, P., and Raaijmakers, J. M. (2013). The rhizosphere microbiome: significance of plant beneficial, plant pathogenic, and human pathogenic microorganisms. FEMS Microbiol. Rev. 37, 634-663. doi: 10.1111/ 1574-6976.12028

Moyne, A.-L., Cleveland, T. E., and Tuzun, S. (2004). Molecular characterization and analysis of the operon encoding the antifungal lipopeptide bacillomycin D. FEMS Microbiol. Lett. 234, 43-49. doi: 10.1111/j.1574-6968.2004.tb09511.x

Niu, J., Chao, J., Xiao, Y., Chen, W., Zhang, C., Liu, X., et al. (2017). Insight into the effects of different cropping systems on soil bacterial community and tobacco bacterial wilt rate. J. Basic Microbiol. 57, 3-11. doi: 10.1002/jobm.201600222 
Niu, J., Rang, Z., Zhang, C., Chen, W., Tian, F., Yin, H., et al. (2016). The succession pattern of soil microbial communities and its relationship with tobacco bacterial wilt. BMC Microbiol. 16:233. doi: 10.1186/s12866-0160845-x

Olesen, J. M., Bascompte, J., Dupont, Y. L., and Jordano, P. (2007). The modularity of pollination networks. Proc. Natl. Acad. Sci. U.S.A. 104, 19891-19896. doi: 10.1073 /pnas.0706375104

Ongena, M., and Jacques, P. (2008). Bacillus lipopeptides: versatile weapons for plant disease biocontrol. Trends Microbiol. 16, 115-125. doi: 10.1016/j.tim.2007. 12.009

Palaniyandi, S. A., Yang, S. H., Zhang, L., and Suh, J.-W. (2013). Effects of actinobacteria on plant disease suppression and growth promotion. Appl. Microbiol. Biotechnol. 97, 9621-9636. doi: 10.1007/s00253-0135206-1

Penton, C. R., Gupta, V. V., Tiedje, J. M., Neate, S. M., Ophel-Keller, K., Gillings, M., et al. (2014). Fungal community structure in disease suppressive soils assessed by 28S LSU gene sequencing. PLOS ONE 9:e93893. doi: 10.1371/ journal.pone.0093893

Pieterse, C. M., and Dicke, M. (2007). Plant interactions with microbes and insects: from molecular mechanisms to ecology. Trends Plant Sci. 12, 564-569. doi: 10.1016/j.tplants.2007.09.004

Poudel, R., Jumpponen, A., Schlatter, D. C., Paulitz, T. C., Gardener, B. B. M., Kinkel, L. L., et al. (2016). Microbiome networks: a systems framework for identifying candidate microbial assemblages for disease management. Phytopathology 106, 1083-1096. doi: 10.1094/PHYTO-02-16-0058-FI

Qiu, M., Zhang, R., Xue, C., Zhang, S., Li, S., Zhang, N., et al. (2012). Application of bio-organic fertilizer can control Fusarium wilt of cucumber plants by regulating microbial community of rhizosphere soil. Biol. Fertil. Soils 48, 807-816. doi: 10.1007/s00374-012-0675-4

Sanguin, H., Sarniguet, A., Gazengel, K., Moenne-Loccoz, Y., and Grundmann, G. L. (2009). Rhizosphere bacterial communities associated with disease suppressiveness stages of take-all decline in wheat monoculture. New Phytol. 184, 694-707. doi: 10.1111/j.1469-8137.2009.03010.x

Santhanam, R., Luu, V. T., Weinhold, A., Goldberg, J., Oh, Y., and Baldwin, I. T. (2015). Native root-associated bacteria rescue a plant from a sudden-wilt disease that emerged during continuous cropping. Proc. Natl. Acad. Sci. U.S.A. 112, E5013-E5020. doi: 10.1073/pnas.1505765112

Schisler, D., Slininger, P., Behle, R., and Jackson, M. (2004). Formulation of Bacillus spp. for biological control of plant diseases. Phytopathology 94, 1267-1271. doi: 10.1094/PHYTO.2004.94.11.1267

Shi, S., Nuccio, E., Herman, D. J., Rijkers, R., Estera, K., Li, J., et al. (2015). Successional trajectories of rhizosphere bacterial communities over consecutive seasons. MBio 6:e00746-15. doi: 10.1128/mBio.00746-15
Shi, S., Nuccio, E. E., Shi, Z. J., He, Z., Zhou, J., and Firestone, M. K. (2016). The interconnected rhizosphere: high network complexity dominates rhizosphere assemblages. Ecol. Lett. 19, 926-936. doi: 10.1111/ele.12630

Soffer, N., Zaneveld, J., and Vega Thurber, R. (2015). Phage-bacteria network analysis and its implication for the understanding of coral disease. Environ. Microbiol. 17, 1203-1218. doi: 10.1111/1462-2920.12553

Tylianakis, J. M., Tscharntke, T., and Lewis, O. T. (2007). Habitat modification alters the structure of tropical host-parasitoid food webs. Nature 445, 202-205. doi: $10.1038 /$ nature 05429

van Elsas, J. D., Chiurazzi, M., Mallon, C. A., Elhottova, D., Kristufek, V., and Salles, J. F. (2012). Microbial diversity determines the invasion of soil by a bacterial pathogen. Proc. Natl. Acad. Sci. U.S.A. 109, 1159-1164. doi: 10.1073/ pnas.1109326109

Wang, R., Zhang, H., Sun, L., Qi, G., Chen, S., and Zhao, X. (2017). Microbial community composition is related to soil biological and chemical properties and bacterial wilt outbreak. Sci. Rep. 7, 343. doi: 10.1038/s41598-017-00472-6

Weller, D. M., Raaijmakers, J. M., Gardener, B. B., and Thomashow, L. S. (2002). Microbial populations responsible for specific soil suppressiveness to plant pathogens. Annu. Rev. Phytopathol. 40, 309-348. doi: 10.1146/annurev.phyto. 40.030402.110010

Yeung, M. S., Tegnér, J., and Collins, J. J. (2002). Reverse engineering gene networks using singular value decomposition and robust regression. Proc. Natl. Acad. Sci. U.S.A. 99, 6163-6168. doi: 10.1073/pnas.092576199

Zhang, Y., Cong, J., Lu, H., Yang, C., Yang, Y., Zhou, J., et al. (2014). An integrated study to analyze soil microbial community structure and metabolic potential in two forest types. PLOS ONE 9:e93773. doi: 10.1371/journal.pone.0093773

Zhou, J., Deng, Y., Luo, F., He, Z., Tu, Q., and Zhi, X. (2010). Functional molecular ecological networks. MBio 1:e00169-10. doi: 10.1128/mBio.00169-10

Zhou, J., Deng, Y., Luo, F., He, Z., and Yang, Y. (2011). Phylogenetic molecular ecological network of soil microbial communities in response to elevated CO2. MBio 2:e0122-11. doi: 10.1128/mBio.00122-11

Conflict of Interest Statement: The authors declare that the research was conducted in the absence of any commercial or financial relationships that could be construed as a potential conflict of interest.

Copyright (C) 2017 Yang, Li, Xiao, Gu, Liu, Liang, Liu, Hu, Meng and Yin. This is an open-access article distributed under the terms of the Creative Commons Attribution License (CC BY). The use, distribution or reproduction in other forums is permitted, provided the original author(s) or licensor are credited and that the original publication in this journal is cited, in accordance with accepted academic practice. No use, distribution or reproduction is permitted which does not comply with these terms. 